\title{
Stability of a vortex street of finite vortices
}

\author{
By P. G. SAFFMAN AND J. C. SCHATZMAN
}

Applied Mathematics, California Institute of Technology, Pasadena, CA 91125, U.S.A.

(Received 2 April 1981 and in revised form 1 September 1981)

The stability of the finite-area Kármán 'vortex street' to two-dimensional disturbances is determined. It is shown that for vortices of finite size there exists a finite range of spacing ratio $\kappa$ for which the array is stable to infinitesimal disturbances. As the vortex size approaches zero, the range narrows to zero width about the classical von Kármán value of $\mathbf{0} \cdot 281$.

\section{Introduction}

The Kármán vortex street is a regular pattern of vortices consisting of two parallel staggered rows, which, for a certain range of Reynolds number, is observed in the wake of two-dimensional blunt bodies placed in a uniform stream. In a previous paper (Saffman \& Schatzman 1981) an inviscid model for the wake flow was described which consists of two rows of staggered vortices of finite size, extending to infinity in both directions. Steady solutions (which propagate relative to the free stream) were found numerically, and their properties were calculated. These describe an infinite array of uniform two-dimensional vortices, consisting of one row of identical vortices of area $A$ and strength $-\Gamma$ with centroids at positions $x=0, \pm l, \pm 2 l, \pm 3 l, \ldots, y=0$, and of a second row of identical vortices of area $A$ and strength $+\Gamma$ with centroids at $x=d, d \pm l, d \pm 2 l, d \pm 3 l, \ldots, y=-h$. The frame of reference is chosen with a uniform flow $U_{\mathrm{s}}$ in the $z$-direction at infinity as in figure 1 so that the vortices are stationary. It is assumed that the flow is inviscid, incompressible, two-dimensional, and, outside the vortices, irrotational. The case $\mu \equiv d / l=0.5$ was mainly considered (for values of $\mu$ other than 0 and 0.5 translating solutions exist but the street does not move parallel to itself; see Rosenhead (1929)). There are then two independent dimensionless parameters $h / l \equiv \kappa$ and $A / l^{2} \equiv \alpha$ which determine the geometry of the street.

This paper discusses the stability of these steady solutions to two-dimensional disturbances. A normal-mode analysis is carried out and the growth rates and frequencies of the modes are calculated for a range of values of the vortex size and separation/ spacing ratio of the street. It is found that finite size can stabilize the street to infinitesimal disturbances. The results for superharmonic disturbances are in accord with those predicted by energy arguments based on Kelvin's variational principle. It will be pointed out that a plausible but non-rigorous attempt to use these energy arguments for subharmonic disturbances leads to fallacious conclusions. 


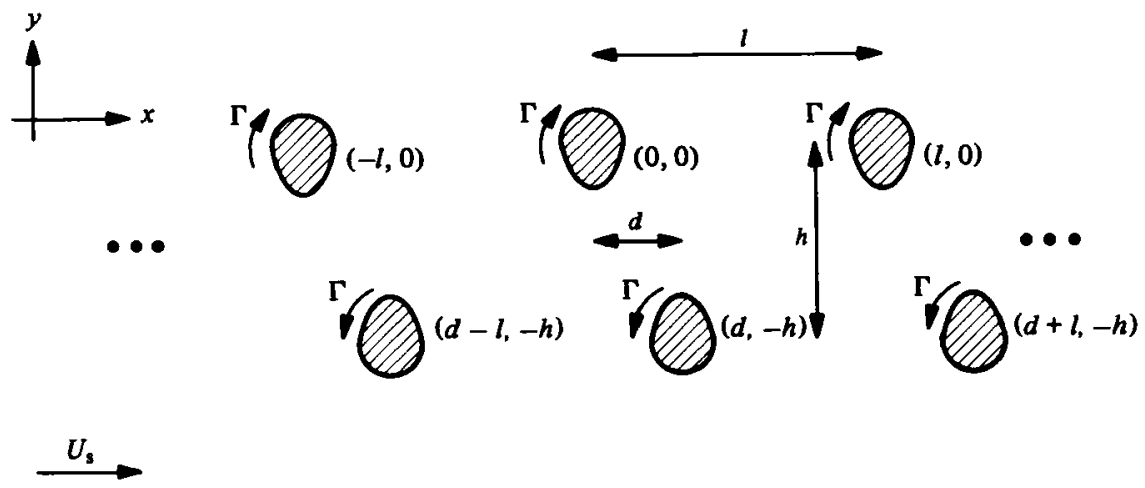

Figure 1. The configuration of the fully infinite vortex street with arbitrary stagger.

\section{Subharmonic Instabilities of the point-vortex array}

The limiting case of point vortices $(\alpha=0)$ was studied by von Kármán (1912), see Lamb $(1932, \S 156)$. It was shown that infinitesimal two-dimensional disturbances of wavelength $l / p$ grow like $e^{\sigma t}$, where

$$
\begin{gathered}
\frac{2 l^{2}}{\pi \Gamma} \sigma= \pm B \pm\left(A^{2}-C^{2}\right)^{\frac{1}{2}} \\
A=2 p(1-p)-\operatorname{sech}^{2} \pi \kappa \\
B=i\left\{\frac{2 p \sinh \pi \kappa(1-2 p)}{\cosh \pi \kappa}+\frac{\sinh 2 \pi \kappa p}{\cosh ^{2} \pi \kappa}\right\} \\
C=\frac{\cosh 2 \pi \kappa p}{\cosh ^{2} \pi \kappa}-\frac{2 p \cosh \pi \kappa(1-2 p)}{\cosh \pi \kappa}
\end{gathered}
$$

Note that $p$ need not be an integer or rational. Since the steady flow has wavelength $l$, it follows from Floquet- or Bloch-wave theory that the normal modes of the system (for finite as well as point vortices) are of the form

$$
e^{\sigma t} e^{2 \pi x p l l} P(x, y) \text {, }
$$

where $P(x+l, y) \equiv P(x, y)$. Disturbances with $p$ equal to an integer or zero will be called superharmonic; they always have wavelength $l$. If $p$ is not equal to an integer, there is clearly no loss of generality in supposing that $0<p<1$, and such disturbances will in general have components with wavelengths greater than $l$ and will be called subharmonic. Note that the properties of these disturbances (including the eigenvalue $\sigma)$ must be the same for $p$ and $1-p$, i.e. the problem exhibits symmetry in $p$ about $p=\mathbf{0} \cdot \mathbf{5}$.

Figure 2 shows the regions of stable $(\mathscr{R} \sigma=0)$ and unstable $(\mathscr{R} \sigma>0)$ eigenfunctions in the $(\kappa, p)$-plane for point vortices. It should be noted that not all eigenfunctions are linearly unstable but for $\kappa \neq \kappa_{\mathrm{c}}$ there always exist unstable disturbances. For $\kappa=\kappa_{\mathrm{c}}$ (where $\cosh ^{2} \pi \kappa_{c}=2$ ) all the disturbances are linearly stable, and this case was identified by von Kármán as the stable configuration of the strect. However, it was discovered by Kochin (1939) that this 'stable' configuration is in fact unstable at second-order approximation in the disturbance amplitude (for an elegant demonstration, see Domm (1956)). These higher-order studies have dealt only with the case 


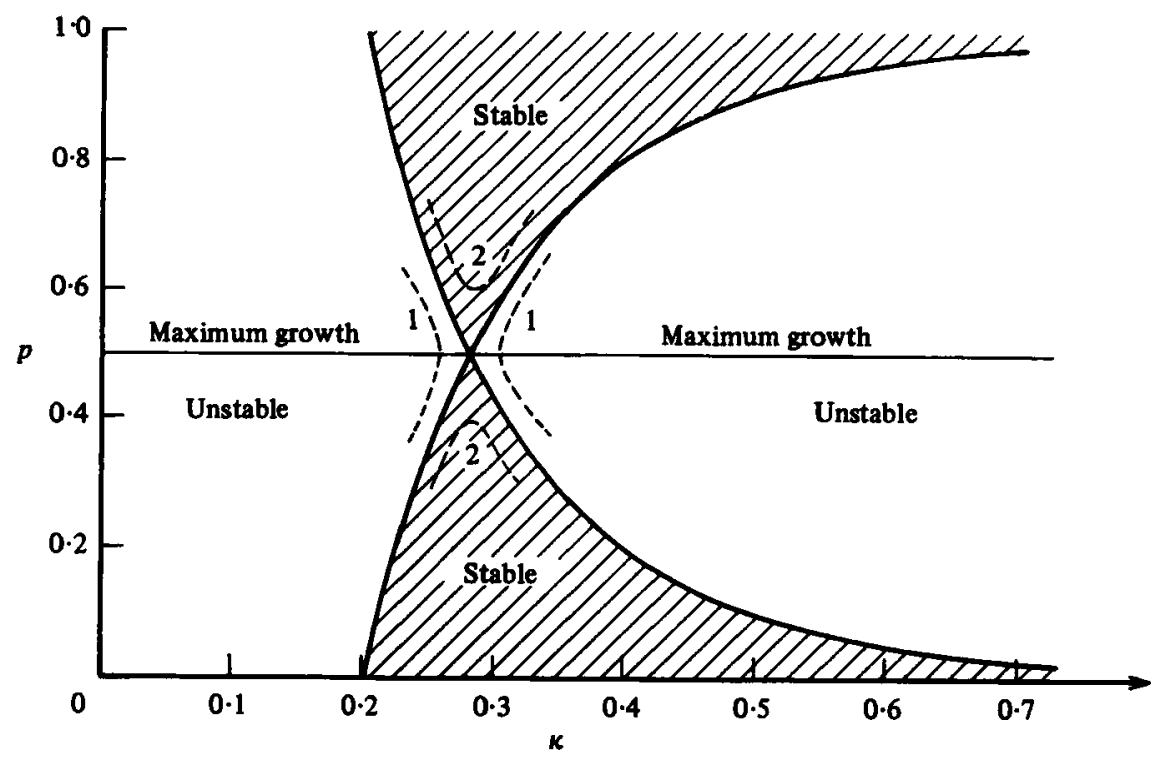

FIGURE 2. The stability boundary in the periodicity-spacing-ratio $(p, \kappa)$-plane for point-vortex configurations. The growth rates are symmetric about $p=0.5$ which gives also the maximum rate of growth for all $\kappa$. Curves 1 and 2 show qualitatively the two possible perturbed boundaries for small area. The calculations indicate that curve 1 is the true result.

$p=0.5$, and it is not known whether approximation of the evolution equations to order higher than first would lead to growing disturbances for other values of $p$. The disturbances for the $p=0.5, \kappa=\kappa_{\mathrm{c}}$ case grow in time as $e^{\epsilon t}$, where $\epsilon>0$ is proportional to the initial disturbance, as opposed to the $e^{\sigma_{R} t}$ behaviour $\left(\sigma_{\mathrm{R}}\right.$ independent of initial conditions) that occurs in the unstable region away from the stability/instability boundary.

Initially, it was hoped that an energy criterion could be used to answer this question of finite-amplitude stability away from the stability boundary. The Kirchhoff-Routh path function $W\left(x_{1}, y_{1}, x_{2}, y_{2}, \ldots\right)$ (which is a measure of the 'interaction energy', see Lin (1943)) determines the motion of the vortices $\left(x_{i}, y_{i}\right)$ through the relations

$$
\frac{d x_{i}}{d t}=-\frac{1}{\Gamma_{i}} \frac{\partial W}{\partial y_{i}}, \quad \frac{d y_{i}}{d t}=\frac{1}{\Gamma_{i}} \frac{\partial W}{\partial x_{i}} .
$$

By a trivial change of variables, say $x_{i} \rightarrow-x_{i}$ for vortices with $\Gamma_{i}=-\Gamma$, this becomes a Hamiltonian system with Hamiltonian $W$. The right-hand sides of the (Hamilton's) equations may be expanded in Taylor series about the steady solutions $x_{i}, y_{i}$. The linearized equations reproduce figure 2. The second- and higher-order terms are, for sufficiently small deviation from the steady state, a small correction to the linear (and integrable) system. There exists a body of theory for such 'nearly integrable' Hamiltonian systems in the literature (see e.g. Chirikov 1979). In general, such systems exhibit the slow-instability phenomenon known as 'Arnol'd diffusion'. Fairly general bounds exist for the average growth rate of this instability (e.g. Nekhoroshev 1971) but these do not appear to provide useful conclusions for the present problem.

The stability boundaries of figure 2 will obviously be perturbed by the effect of finite size of the vortices, and the degenerate saddle will separate into one of the 


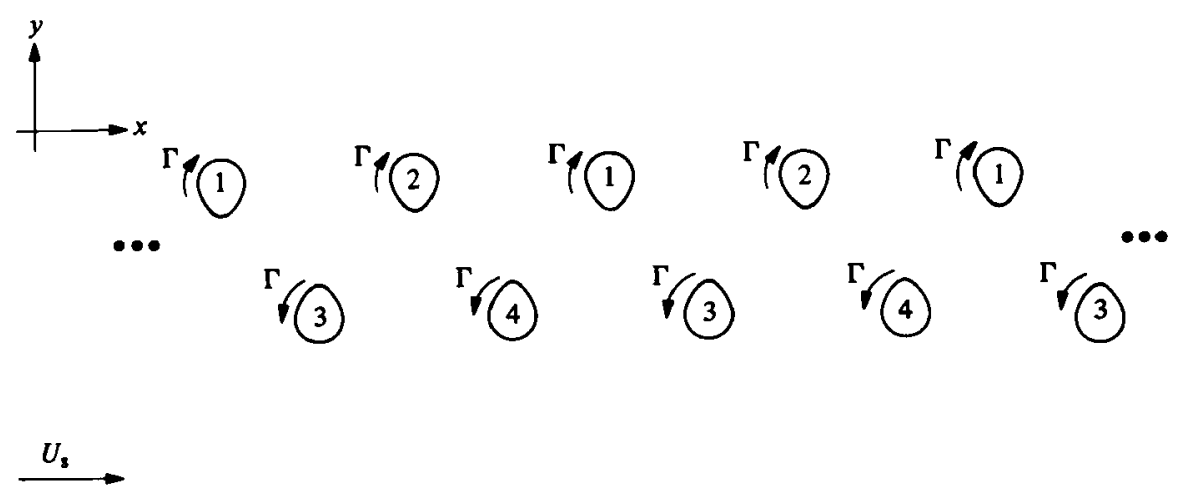

Fiaure 3. Diagram of the vortex-street configuration showing the four independent perturbations.

possibilities marked by the dashed lines in the figure. If case 1 is the situation, then there will be stability to infinitesimal disturbances for a finite range of $k$ in the vicinity of $\kappa=\kappa_{\mathrm{c}}$. If case 2 obtains, then finite vortex size makes the array unstable for all $\kappa$. Remember that the symmetry about $p=0.5$ is valid for all $\alpha$, so cases 1 and 2 are the only possibilities.

In principle, the problem of deciding between case 1 and case 2 can be treated by perturbation theory by expanding in powers of the area of the vortices, i.e. $\alpha$. However, for reasons to be given below, it appears that the algebraic complexity is great, and direct numerical methods were employed instead. These, of course, have the advantage that they give results for finite areas not accessible to perturbation methods. In order to decide between case 1 and case 2 , it clearly suffices because of the symmetry of figure 2 to consider only the subharmonic (pairing) disturbances with $p=0.5$, and we therefore restrict attention henceforth to disturbances that are periodic with period $2 l$ in the $x$-direction. Of course, there is no guarantee that $p=0.5$ gives the maximum growth rate for finite $\alpha$, so that the stability boundary for moderate and large $\alpha$ determined with $p=0.5$ may not be accurate. Note that the superharmonic disturbances are then automatically included, as these are trivially of period $2 l$.

\section{Analysis of the stability}

It is appropriate for disturbances with period $2 l$ to consider four independent perturbed vortex shapes (and positions), corresponding to the four vortices in one period $2 l$, and extended periodically to infinity along the street, as in figure 3 . The approach is to calculate the first variation of the velocity field due to a perturbation in vortex shape and position, and then to require that the linearized kinematic condition be satisfied on the boundaries of the vortices. In particular, solutions are looked for that are normal modes proportional to $e^{\sigma t}$; an eigenvalue problem is the result.

A convenient parametrization for the vortex boundaries is a polar co-ordinate representation

$$
\left.\begin{array}{rl}
z(\theta) & =z_{0}(\theta)+z^{\prime}(\theta), \\
z^{\prime}(\theta) & =\left[\frac{1}{2} \alpha_{0}+\sum_{n=1}^{N}\left(a_{n} \cos n \theta+b_{n} \sin n \theta\right)\right] e^{i \theta} .
\end{array}\right\}
$$


Here $z_{0}(\theta)$ describes the steady boundary in question and $z^{\prime}(\theta)$ describes the added disturbances.

As was shown previously (Saffman \& Schatzman 1981), the complex velocity field produced by a single row of vortices (of spacing $2 l$ ) can be calculated by integration around the boundary of a single vortex in the row as follows:

$$
u+i v=\frac{\Gamma}{2 \pi A} \int \log \left|\sin \frac{\pi}{l}(z-Z)\right| d Z,
$$

where $z \equiv z+i y$ is the complex co-ordinate, lower-case variables refer to the point of evaluation of the flow field and capital variables refer to the path of integration. When evaluated on a vortex boundary, this will give the velocity contribution on the vortex boundaries of each vortex in the corresponding $2 l$-periodic row due to disturbances of the other three $2 l$-periodic vortex rows, added to the unperturbed value. Substituting $z=z_{0}+z^{\prime}, Z=Z_{0}+Z^{\prime}$, and assuming constant area $A$, the corresponding first variation of the velocity contribution is

$$
u^{\prime}+i v^{\prime}=\frac{\Gamma}{2 \pi A} \int\left[\log \left|\sin \frac{\pi}{2 l}\left(z_{0}-Z_{0}\right)\right| \frac{d Z^{\prime}}{d \Theta}+\mathscr{R}\left\{\frac{\pi}{2 l} \cot \frac{\pi}{2 l}\left(z_{0}-Z_{0}\right)\left(z^{\prime}-Z^{\prime}\right)\right\} \frac{d Z_{0}}{d \Theta}\right] d \Theta .
$$

As was remarked in the previous paper, the 'self-induced' velocity for one row of vortices may be written:

$$
\left.u+i v=\int\left[\log \left|\frac{\sin \frac{\pi}{2 l}(z-Z)}{\frac{\pi}{2 l}(z-Z)}\right|-i\left[\arg (z-Z)-\frac{1}{2} \Theta\right]\right\} \frac{d Z}{d \Theta}+\frac{1}{2} i Z\right] d \Theta-i \pi z,
$$

where the arg function is taken so as to make the integrand periodic. The corresponding first variation is then:

$$
\begin{aligned}
u^{\prime}+i v^{\prime} & =\frac{\Gamma}{2 \pi A} \int\left\{\left[\mathscr{R}\left\{\left[\frac{\pi}{2 l} \cot \frac{\pi}{2 l}\left(z_{0}-Z_{0}\right)-\frac{1}{z_{0}-Z_{0}}\right]\left(z^{\prime}-Z^{\prime}\right)\right\}-\mathscr{I}\left(\frac{z^{\prime}-Z^{\prime}}{z_{0}-Z_{0}}\right)\right] \frac{d Z_{0}}{d \Theta}\right. \\
+ & {\left.\left[\log \left|\frac{\sin \frac{\pi}{2 l}\left(z_{0}-Z_{0}\right)}{\frac{\pi}{2 l}\left(z_{0}-Z_{0}\right)}\right|-i\left[\arg \left(z_{0}-Z_{0}\right)-\frac{1}{2} \Theta\right]\right] \frac{d Z^{\prime}}{d \Theta}+\frac{1}{2} i Z^{\prime}\right\} d \Theta-i \pi z^{\prime} }
\end{aligned}
$$

Note that all singularities of the integrand have been removed. To calculate the change in flow field due to the complete disturbance, three terms of the form (3.3) and one of the form (3.5) are summed with appropriate choice for the sign of $\Gamma$ and the vortex co-ordinate parameters in each case.

The kinematic condition that the vortex boundaries move with the fluid may be written

Here

$$
\frac{D}{D t}[r-R(\theta, t)]=0 .
$$

$$
\left.\begin{array}{rl}
R(\theta, t) & \equiv R_{0}(\theta)+R^{\prime}(\theta, t) \\
\frac{D}{D t} & \equiv \frac{\partial}{\partial t}+u_{r} \frac{\partial}{\partial r}+\frac{1}{r} u_{\theta} \frac{\partial}{\partial \theta}
\end{array}\right\}
$$


where, as before, the subscript zero refers to the unperturbed quantity and the prime refers to added perturbations. Also, $u_{r}$ and $u_{\theta}$ are the polar velocity components. The solutions of interest are normal modes with perturbations proportional to $e^{\sigma t}$, so that

$$
R^{\prime}(\theta, t)=e^{\sigma t} R^{\prime}(\theta), \quad u(r, \theta, t)=u_{0}(r, \theta)+e^{\sigma t} u^{\prime}(r, \theta),
$$

where the latter holds for each velocity component. To leading order

$$
u(r, \theta)=\left[u_{0}+e^{\sigma t}\left(R^{\prime} \frac{\partial u_{0}}{\partial r}+u^{\prime}\right)\right]\left(R_{0}, \theta\right)
$$

for each velocity component. Equations (3.7)-(3.9) may be substituted into (3.6) and terms of second and higher order in the perturbation omitted, giving

$$
u_{r}^{\prime}+\frac{\partial u_{r 0}}{\partial r} R^{\prime}-\frac{1}{R_{0}} \frac{d R_{0}}{\partial \theta}\left(u_{\theta}^{\prime}+\frac{\partial u_{\theta 0}}{\partial r} R^{\prime}\right)=\sigma R^{\prime} \frac{1}{R_{0}^{2}} \frac{d R_{0}}{d \theta} u_{\theta 0} R^{\prime}+\frac{u_{\theta 0}}{R_{0}} \frac{d R^{\prime}}{d \theta},
$$

where now all quantities are evaluated on $r=R_{0}(\theta)$, and use has been made of the fact that

$$
u_{r 0}-\frac{1}{R_{0}} \frac{d R_{0}}{d \theta} u_{\theta 0}=0 \text { on } \quad r=R_{0} .
$$

The left-hand side of (3.10) is the perturbation in normal velocity component $\delta u_{n}$ divided by the geometric factor

$$
\cos \eta \equiv\left[1+\left(\frac{1}{R_{0}} \frac{d R_{0}}{d \theta}\right)^{2}\right]^{-\frac{1}{2}}
$$

where the effect of the change in normal direction due to the perturbation has been omitted. Also, $u_{\theta 0}$ and the unperturbed tangential velocity component $u_{t 0}$ are related by

so that (3.10) may be written

$$
u_{\theta 0}=u_{\mathrm{t} 0} \cos \eta
$$

$$
\delta u_{\mathrm{n}}=\sigma \cos \eta R^{\prime}-\cos ^{2} \eta \frac{1}{R_{0}^{2}} \frac{d R_{0}}{d \theta} u_{\mathrm{t} 0} R^{\prime}+\cos ^{2} \eta \frac{u_{\mathrm{t} 0}}{R_{0}} \frac{d R^{\prime}}{d \theta} .
$$

This may be equated to the corresponding quantity calculated by the integration above (i.e. by (3.3) and (3.5)) and using

$$
\left.\begin{array}{c}
\delta u_{\mathrm{n}}=\cos \eta\left(u_{r}^{\prime}-\frac{1}{R_{0}} \frac{d R_{0}}{d \theta} u_{\theta}^{\prime}\right), \\
\cos \theta+v^{\prime} \sin \theta, \quad u_{\theta}^{\prime}=-u^{\prime} \sin \theta+v^{\prime} \cos \theta .
\end{array}\right\}
$$

After substituting (3.1) and evaluating at the $2 N+1$ points

$$
\theta=\theta_{j} \equiv \frac{2 \pi j}{2 N+1} \quad(j=0,1, \ldots, 2 N)
$$

the result is a generalized eigenvalue problem of the form

$$
\mathbf{A w}=\sigma \mathbf{B w},
$$


where $A$ and $B$ are $8 N+4$ by $8 N+4$ matrices and $w$ is a vector containing the four sets of $2 N+1$ Fourier coefficients from (3.1). The real parts of the eigenvalues of this system then give the growth rates of the corresponding normal modes. Inspection leads to the conclusion that, if $\sigma$ is an eigenvalue, then so are $-\sigma, \sigma^{*}$, and $-\sigma^{*}$. Thus, it is evident that, at best, a steady state may have only normal modes with zero linear growth rate, and otherwise the state is linearly unstable.

For numerical purposes, the system (3.17) may be simplified somewhat by recognizing the symmetry of the problem. It is sufficient to consider disturbances where the vortex-shape perturbations $z^{\prime}$ are negative for vortices of rows 1 and 2 above, and similarly for rows 3 and 4 . This simplification reduces the size of the system to $4 N+2$ equations in two sets of Fourier coefficients. Values of $N$ from 10 to 25 were used, depending on the size of the vortices.

Computations of the eigensystem were performed using the EIGZF subroutine in the IMSL library on an IBM 3032 computer (64 bit floating point). Some computational difficulties were encountered; an explanation follows. Note that, for isolated circular vortices of area $A$ and circulation $\Gamma$, normal-mode perturbations exist of the form (see Lamb 1932, pp. 230-1)

$$
R^{\prime}=\epsilon \cos m \theta e^{\sigma t}, \quad \sigma=\frac{1}{2} i \frac{\Gamma}{2 \pi A}(m-1) \quad(m=2,3,4, \ldots) .
$$

Since the corresponding flow-field perturbations fall off in distance $r$ as $r^{-m-1}$, and since small-area vortices for the street are nearly circular, it is evident that, for small $A$ and large $m$, there will exist such solutions for the street. These in fact are the superharmonic disturbances. For small area $A$, these eigenvalues are much larger in modulus than the subharmonic $(p=0.5)$ modes, which are bounded as $A$ decreases. In fact, although this behaviour is most severe for small areas, for all states that were computed, these 'nearly isolated' modes had eigenvalues with dominating moduli. This characteristic showed itself in a great sensitivity of the calculated small eigenvalues to errors in the steady calculations (hence the matrices $\mathbf{A}$ and $\mathbf{B}$ ). Typically, an error of roughly $0.1 \%$ in radius or tangential velocity completely destroyed the small-eigenvalue computation. A second computational problem was failure of convergence of the iterative procedure for eigenvalue and eigenvector computation. This difficulty usually manifested itself near the stability/instability interface, and the explanation is not clear.

The results of the stability calculations are tabulated in table 1 and summarized in figure 4. Outside of the indicated region in the $(\kappa, \alpha)$-plane, there are modes with positive growth rates, and hence these states are linearly unstable. Inside this region, there are no such modes (with $x$-period $2 l$ ). With the exception of the smaller-energy state in the non-unique region, all eigenvalues corresponding to superharmonic disturbances were found to be purely imaginary. Aside from the trivial modes (uniform displacements) all non-growing modes are found to be given by (3.18) approximately with best matching for large $m$. There is agreement with the unsteady initial-value calculations of Christiansen \& Zabusky (1973), as indicated in the figure. The superharmonic disturbances were always stable except for the smaller-energy solution in the nonunique region between curves 1 and 2 .

For small areas, the width in $\kappa$ of the stability region decreases and a plot of calculations in the vicinity (figure 5) indicates that the critical value of area $\alpha$ at which 


\begin{tabular}{|c|c|c|c|c|c|}
\hline $\boldsymbol{\kappa}$ & $\frac{A}{l^{2}}$ & $\frac{l}{\Gamma} U_{\mathrm{s}}$ & $\frac{l}{\Gamma^{2}} T$ & $\frac{l^{2}}{\Gamma}\left|\sigma_{\mathrm{R}}\right|$ & $\frac{l^{2}}{\Gamma}\left|\sigma_{I}\right|$ \\
\hline 0.2 & $\begin{array}{l}0 \\
0 \cdot 49141(-3) \\
0 \cdot 43903(-1) \\
0 \cdot 69626(-1) \\
0 \cdot 93758(-1) \\
0 \cdot 13126 \\
0 \cdot 18944 \\
0 \cdot 26522\end{array}$ & $\begin{array}{l}0 \cdot 278447 \\
0 \cdot 27845 \\
0 \cdot 27586 \\
0 \cdot 27204 \\
0 \cdot 26704 \\
0.25709 \\
0.23839 \\
0 \cdot 21281\end{array}$ & $\begin{array}{l}\infty \\
0.58448 \\
0.22718 \\
0.19081 \\
0.16758 \\
0.14180 \\
0.11485 \\
0.43904(-1)\end{array}$ & $\begin{array}{l}0.29825 \\
0 \cdot 2983 \\
0.2996 \\
0 \cdot 3003 \\
0 \cdot 3000 \\
0 \cdot 2959 \\
0 \cdot 2732 \\
0 \cdot 1007\end{array}$ & $\begin{array}{l}0.72656 \\
0.7266 \\
0.7113 \\
0.6886 \\
0.6592 \\
0.5999 \\
0.4797 \\
0.2672\end{array}$ \\
\hline 0.23 & $\begin{array}{l}0 \\
0 \cdot 78551(-4) \\
0 \cdot 54099(-1) \\
0 \cdot 11189 \\
0 \cdot 16503 \\
0 \cdot 19545 \\
0 \cdot 23587 \\
0 \cdot 29187\end{array}$ & $\begin{array}{l}0.309248 \\
0 \cdot 30925 \\
0 \cdot 30609 \\
0 \cdot 29933 \\
0 \cdot 28125 \\
0 \cdot 27142 \\
0 \cdot 25755 \\
0 \cdot 23803\end{array}$ & $\begin{array}{l}\infty \\
0.73921 \\
0.21939 \\
0.17425 \\
0.13271 \\
0.12026 \\
0.10691 \\
0.92648(-1)\end{array}$ & $\begin{array}{l}0 \cdot 18451 \\
0 \cdot 1845 \\
0 \cdot 1890 \\
0 \cdot 1961 \\
0 \cdot 1948 \\
0 \cdot 1882 \\
0 \cdot 1603 \\
0 \cdot 8594(-1)\end{array}$ & $\begin{array}{l}0.76342 \\
0.7634 \\
0.7467 \\
0.6950 \\
0.6242 \\
0.5754 \\
0.4951 \\
0.5671\end{array}$ \\
\hline 0.25 & $\begin{array}{l}0 \\
0 \cdot 78549(-4) \\
0 \cdot 57877(-1) \\
0 \cdot 99641(-1) \\
0 \cdot 10711 \\
0 \cdot 11506 \\
0 \cdot 19170 \\
0 \cdot 20770 \\
0 \cdot 22598 \\
0 \cdot 24714 \\
0 \cdot 27187\end{array}$ & $\begin{array}{l}0.327897 \\
0.32790 \\
0.32490 \\
0.31889 \\
0.31748 \\
0.31587 \\
0.29544 \\
0.29032 \\
0.28425 \\
0.27700 \\
0.26835\end{array}$ & $\begin{array}{l}\infty \\
0 \cdot 74559 \\
0 \cdot 22036 \\
0 \cdot 17752 \\
0 \cdot 17187 \\
0 \cdot 16628 \\
0 \cdot 12734 \\
0 \cdot 12144 \\
0 \cdot 11535 \\
0 \cdot 10900 \\
0 \cdot 10241\end{array}$ & $\begin{array}{l}0.10985 \\
0.1099 \\
0.1154 \\
0 \cdot 1201 \\
0.1204 \\
0 \cdot 1202 \\
0.7787(-1) \\
0 \cdot 1709(-1) \\
0 \\
0.2075 \\
0.2384\end{array}$ & $\begin{array}{l}0.77768 \\
0.7777 \\
0.7632 \\
0.7360 \\
0.7300 \\
0.7232 \\
0.6522 \\
0.6427 \\
0.5304 / 0.7896 \\
0.7554 \\
0.6442\end{array}$ \\
\hline 0.26 & $\begin{array}{l}0 \\
0 \cdot 12059 \\
0 \cdot 15874 \\
0 \cdot 16438 \\
0 \cdot 17028 \\
0 \cdot 21258\end{array}$ & $\begin{array}{l}0 \cdot 336666 \\
0 \cdot 32466 \\
0 \cdot 31581 \\
0 \cdot 31432 \\
0 \cdot 31273 \\
0 \cdot 30011\end{array}$ & $\begin{array}{l}\infty \\
0.16583 \\
0.14461 \\
0.14195 \\
0.13927 \\
0.12270\end{array}$ & $\begin{array}{l}0 \cdot 73237(-1) \\
0 \cdot 7640(-1) \\
0 \cdot 4205(-1) \\
0 \cdot 2596(-1) \\
0 \\
0\end{array}$ & $\begin{array}{l}0.78198 \\
0.7316 \\
0.7026 \\
0.6986 \\
0.6688 / 0.7204 \\
0.5357 / 0.8620\end{array}$ \\
\hline 0.27 & $\begin{array}{l}0 \\
0 \cdot 78547(-4) \\
0.52228(-1) \\
0 \cdot 11023 \\
0 \cdot 11787 \\
0 \cdot 12595 \\
0 \cdot 20142 \\
0 \cdot 21661 \\
0 \cdot 23373 \\
0.25327\end{array}$ & $\begin{array}{l}0.345072 \\
0.34507 \\
0.34315 \\
0.33612 \\
0.33479 \\
0.33328 \\
0.31468 \\
0.31012 \\
0.30473 \\
0.29833\end{array}$ & $\begin{array}{l}\infty \\
0.75232 \\
0.23519 \\
0.17615 \\
0.17091 \\
0.16573 \\
0.12977 \\
0.12437 \\
0.11880 \\
0.11302\end{array}$ & $\begin{array}{l}0.37231(-1) \\
0 \cdot 3723(-1) \\
0.4078(-1) \\
0 \cdot 2624(-1) \\
0.6564(-2) \\
0 \\
0 \\
0 \cdot 1387 \\
0 \cdot 2870 \\
0 \cdot 3419\end{array}$ & $\begin{array}{l}0.78452 \\
0.7845 \\
0.7760 \\
0.7487 \\
0.7663 \\
0.7162 / 0 \cdot 7622 \\
0.5489 / 0.8941 \\
0 \cdot 1043(+1) \\
0.9221 \\
0.8028\end{array}$ \\
\hline 0.28054 & $\begin{array}{l}0 \\
0.78546(-4) \\
0.28358(-2) \\
0.38640(-2) \\
0.40889(-2) \\
0.43203(-2) \\
0.50531(-2)\end{array}$ & $\begin{array}{l}0 \cdot 353646 \\
0 \cdot 35355 \\
0 \cdot 35354 \\
0 \cdot 35354 \\
0 \cdot 35354 \\
0 \cdot 35354 \\
0 \cdot 35353\end{array}$ & $\begin{array}{l}\infty \\
0.75600 \\
0.47061 \\
0.44599 \\
0.44148 \\
0.43710 \\
0.42464\end{array}$ & $\begin{array}{l}0.34637(-4) \\
0.3369(-4) \\
0.3920(-4) \\
0.2533(-4) \\
0.1516(-4) \\
0 \\
0\end{array}$ & $\begin{array}{l}0.78540 \\
0 \cdot 7854 \\
0 \cdot 7854 \\
0 \cdot 7854 \\
0 \cdot 7854 \\
0 \cdot 7853 / 0 \cdot 7854 \\
0 \cdot 7853 / 0 \cdot 7854\end{array}$ \\
\hline $0 \cdot 280549$ & $\begin{array}{l}0 \\
0.78546(-4) \\
0.70738(-3)\end{array}$ & $\begin{array}{l}0 \cdot 353553 \\
0 \cdot 35355 \\
0 \cdot 35355\end{array}$ & $\begin{array}{l}\infty \\
0.75601 \\
0.58110\end{array}$ & $\begin{array}{l}0.32318(-5) \\
0.2047(-5) \\
0.3719(-5)\end{array}$ & $\begin{array}{l}0.78540 \\
0.7854 \\
0.7854\end{array}$ \\
\hline
\end{tabular}




\begin{tabular}{|c|c|c|c|c|c|}
\hline$\kappa$ & $\frac{A}{l^{2}}$ & $\frac{l}{\Gamma} U$ & $\frac{l}{\Gamma^{2}} T$ & $\frac{l^{2}}{\Gamma}\left|\sigma_{\mathrm{B}}\right|$ & $\frac{l^{2}}{\Gamma}\left|\sigma_{1}\right|$ \\
\hline & $\begin{array}{l}0.11355(-2) \\
0.12583(-2) \\
0.13875(-2) \\
0.28358(-2)\end{array}$ & $\begin{array}{l}0.35355 \\
0.35355 \\
0.35355 \\
0.35355\end{array}$ & $\begin{array}{l}0.54345 \\
0.53527 \\
0.52750 \\
0.47061\end{array}$ & $\begin{array}{l}0 \cdot 2774(-5) \\
0 \cdot 1327(-5) \\
0 \\
0\end{array}$ & $\begin{array}{l}0.7854 \\
0.7854 \\
0.7854 / 0.7854 \\
0.7854 / 0 \cdot 7854\end{array}$ \\
\hline 0.280549926 & $\begin{array}{l}0 \\
0 \cdot 78546(-4) \\
0 \cdot 38640(-2) \\
0 \cdot 26145(-1) \\
0 \cdot 47515(-1) \\
0 \cdot 76562(-1) \\
0 \cdot 11525 \\
0 \cdot 20468 \\
0 \cdot 21914 \\
0 \cdot 23527 \\
0 \cdot 25349\end{array}$ & $\begin{array}{l}0 \cdot 353553 \\
0 \cdot 35355 \\
0 \cdot 35355 \\
0 \cdot 35315 \\
0 \cdot 35218 \\
0 \cdot 34988 \\
0 \cdot 34493 \\
0 \cdot 32524 \\
0 \cdot 32113 \\
0 \cdot 31630 \\
0 \cdot 31059\end{array}$ & $\begin{array}{l}\infty \\
0 \cdot 75601 \\
0 \cdot 44599 \\
0 \cdot 29386 \\
0 \cdot 24636 \\
0 \cdot 20852 \\
0 \cdot 17625 \\
0 \cdot 13194 \\
0 \cdot 12685 \\
0 \cdot 12160 \\
0 \cdot 11617\end{array}$ & $\begin{array}{l}0 \\
0 \\
0 \\
0 \\
0 \\
0 \\
0 \\
0 \\
0 \cdot 2241 \\
0 \cdot 3334 \\
0 \cdot 3837\end{array}$ & $\begin{array}{l}0 \cdot 78540 \\
0 \cdot 78540 / 0.78540 \\
0 \cdot 78531 / 0 \cdot 78541 \\
0 \cdot 7812 / 0 \cdot 7860 \\
0 \cdot 7715 / 0 \cdot 7878 \\
0 \cdot 7487 / 0 \cdot 7926 \\
0 \cdot 7022 / 0 \cdot 8056 \\
0 \cdot 5406 / 0 \cdot 9917 \\
0 \cdot 1066(+1) \\
0.9525 \\
0.8395\end{array}$ \\
\hline 0.280551 & $\begin{array}{l}0 \\
0 \cdot 78546(-4) \\
0 \cdot 61615(-3) \\
0 \cdot 70738(-3) \\
0 \cdot 80493(-3) \\
0 \cdot 28358(-2)\end{array}$ & $\begin{array}{l}0 \cdot 353553 \\
0 \cdot 35355 \\
0 \cdot 35355 \\
0 \cdot 35355 \\
0 \cdot 35355 \\
0 \cdot 35355\end{array}$ & $\begin{array}{l}\infty \\
0.79152 \\
0.59209 \\
0.58110 \\
0.57082 \\
0.47061\end{array}$ & $\begin{array}{l}0.37471(-5) \\
0.4499(-5) \\
0 \cdot 2617(-5) \\
0.1973(-5) \\
0 \\
0\end{array}$ & $\begin{array}{l}0.78540 \\
0.7854 \\
0.7854 \\
0.7854 \\
0.7854 / 0.7854 \\
0.7854 / 0.7854\end{array}$ \\
\hline 0.28056 & $\begin{array}{l}0 \\
0 \cdot 78546(-4) \\
0 \cdot 70738(-3) \\
0 \cdot 21285(-2) \\
0 \cdot 22957(-2) \\
0 \cdot 24694(-2) \\
0 \cdot 50531(-2)\end{array}$ & $\begin{array}{l}0 \cdot 353561 \\
0 \cdot 35356 \\
0 \cdot 35356 \\
0 \cdot 35356 \\
0 \cdot 35356 \\
0 \cdot 35356 \\
0 \cdot 35355\end{array}$ & $\begin{array}{l}\infty \\
0.75601 \\
0.58111 \\
0.49345 \\
0.48743 \\
0.48162 \\
0.42464\end{array}$ & $\begin{array}{l}0.35152(-4) \\
0.3622(-4) \\
0.3412(-4) \\
0 \cdot 1970(-4) \\
0.1433(-4) \\
0 \\
0\end{array}$ & $\begin{array}{l}0.78540 \\
0.7854 \\
0.7854 \\
0.7854 \\
0.7854 \\
0.7854 / 0.7854 \\
0.7852 / 0.7854\end{array}$ \\
\hline 0.29 & $\begin{array}{l}0 \\
0 \cdot 78546(-4) \\
0 \cdot 35769(-1) \\
0 \cdot 70386(-1) \\
0 \cdot 75698(-1) \\
0 \cdot 81253(-1) \\
0 \cdot 12807 \\
0 \cdot 20841 \\
0 \cdot 22227 \\
0 \cdot 25475\end{array}$ & $\begin{array}{l}0 \cdot 360821 \\
0 \cdot 36082 \\
0 \cdot 36016 \\
0 \cdot 35814 \\
0 \cdot 35770 \\
0 \cdot 35721 \\
0 \cdot 35135 \\
0 \cdot 33437 \\
0 \cdot 33065 \\
0 \cdot 32119\end{array}$ & $\begin{array}{l}\infty \\
0 \cdot 75938 \\
0 \cdot 27230 \\
0 \cdot 21853 \\
0 \cdot 21271 \\
0 \cdot 20715 \\
0 \cdot 17127 \\
0 \cdot 13370 \\
0 \cdot 12887 \\
0 \cdot 11880\end{array}$ & $\begin{array}{l}0.32620(-1) \\
0.3262(-1) \\
0.3012(-1) \\
0 \cdot 1559(-1) \\
0.7420(-2) \\
0 \\
0 \\
0 \\
0 \cdot 2752 \\
0.4149\end{array}$ & $\begin{array}{l}0 \cdot 78472 \\
0 \cdot 7847 \\
0 \cdot 7820 \\
0 \cdot 7744 \\
0 \cdot 7729 \\
0 \cdot 7578 / 0 \cdot 7845 \\
0 \cdot 6867 / 0 \cdot 8222 \\
0 \cdot 5395 / 0 \cdot 1109(+1) \\
0 \cdot 1077(+1) \\
0 \cdot 8651\end{array}$ \\
\hline $0 \cdot 3$ & $\begin{array}{l}0 \\
0 \cdot 49106(-3) \\
0 \cdot 80251(-1) \\
0 \cdot 10434 \\
0 \cdot 11102 \\
0 \cdot 11800 \\
0 \cdot 14929 \\
0 \cdot 19894 \\
0 \cdot 21098 \\
0 \cdot 22404 \\
0 \cdot 25413\end{array}$ & $\begin{array}{l}0 \cdot 368179 \\
0 \cdot 36818 \\
0 \cdot 36519 \\
0 \cdot 36295 \\
0 \cdot 36220 \\
0 \cdot 36136 \\
0 \cdot 35683 \\
0 \cdot 34708 \\
0 \cdot 34427 \\
0 \cdot 34104 \\
0 \cdot 33292\end{array}$ & $\begin{array}{l}\infty \\
0 \cdot 61717 \\
0 \cdot 21175 \\
0 \cdot 10998 \\
0 \cdot 18608 \\
0 \cdot 18127 \\
0 \cdot 16283 \\
0 \cdot 14064 \\
0 \cdot 13617 \\
0 \cdot 13163 \\
0 \cdot 12225\end{array}$ & $\begin{array}{l}0.66325(-1) \\
0.6630(-1) \\
0.5236(-1) \\
0 \cdot 3071(-1) \\
0 \cdot 1480(-1) \\
0 \\
0 \\
0 \\
0 \cdot 9528(-1) \\
0 \cdot 2978 \\
0 \cdot 4401\end{array}$ & $\begin{array}{l}0.78259 \\
0 \cdot 7826 \\
0 \cdot 7721 \\
0 \cdot 7656 \\
0 \cdot 7637 \\
0 \cdot 7358 / 0 \cdot 7878 \\
0 \cdot 6699 / 0 \cdot 8396 \\
0 \cdot 5739 / 0 \cdot 9919 \\
0 \cdot 1195(+1) \\
0 \cdot 1097(+1) \\
0.9030\end{array}$ \\
\hline $0 \cdot 31$ & $\begin{array}{l}0 \\
0 \cdot 78544(-4) \\
0.50667(-1)\end{array}$ & $\begin{array}{l}0 \cdot 375205 \\
0 \cdot 37521 \\
0 \cdot 37427\end{array}$ & $\begin{array}{l}\infty \\
0 \cdot 76675 \\
0 \cdot 25196\end{array}$ & $\begin{array}{l}0.99142(-1) \\
0.9914(-1) \\
0.9568(-1)\end{array}$ & $\begin{array}{l}0.77912 \\
0.7791 \\
0.7758\end{array}$ \\
\hline
\end{tabular}




\begin{tabular}{|c|c|c|c|c|c|}
\hline$\kappa$ & $\frac{A}{l^{8}}$ & $\frac{l}{\Gamma} U_{.}$ & $\frac{l}{\Gamma^{2}} T$ & $\frac{l^{2}}{\Gamma}\left|\sigma_{B}\right|$ & $\frac{l^{2}}{\Gamma}\left|\sigma_{I}\right|$ \\
\hline & $\begin{array}{l}0.12276 \\
0.13757 \\
0.14546 \\
0.20165 \\
0.23845 \\
0.25286\end{array}$ & $\begin{array}{l}0.36896 \\
0.36718 \\
0.36613 \\
0.35651 \\
0.34827 \\
0.34466\end{array}$ & $\begin{array}{l}0 \cdot 18181 \\
0 \cdot 17285 \\
0 \cdot 16848 \\
0 \cdot 14312 \\
0 \cdot 13039 \\
0 \cdot 12600\end{array}$ & $\begin{array}{l}0.6074(-1) \\
0.3000(-1) \\
0 \\
0 \\
0.3926 \\
0.4485\end{array}$ & $\begin{array}{l}0.7624 \\
0.7600 \\
0.7270 / 0.7915 \\
0.5917 / 0.1003(+1) \\
0.1030(+1) \\
0.9418\end{array}$ \\
\hline 0.32 & $\begin{array}{l}0 \\
0 \cdot 14979 \\
0 \cdot 16285 \\
0 \cdot 16972 \\
0 \cdot 21245 \\
0 \cdot 23075\end{array}$ & $\begin{array}{l}0 \cdot 381908 \\
0 \cdot 37391 \\
0 \cdot 37224 \\
0 \cdot 37129 \\
0 \cdot 36426 \\
0 \cdot 36066\end{array}$ & $\begin{array}{l}\infty \\
0 \cdot 16988 \\
0 \cdot 16333 \\
0 \cdot 16010 \\
0 \cdot 14273 \\
0 \cdot 13642\end{array}$ & $\begin{array}{l}0 \cdot 1310 \\
0 \cdot 7580(-1) \\
0 \cdot 1929(-1) \\
0 \\
0 \\
0 \cdot 3310\end{array}$ & $\begin{array}{l}0.7744 \\
0.7607 \\
0.7357 \\
0.7259 / 0.8019 \\
0 \cdot 6011 / 0.1101(+1) \\
0 \cdot 1107(+1)\end{array}$ \\
\hline 0.33 & $\begin{array}{l}0 \\
0.78542(-4) \\
0.11194 \\
0.16992 \\
0.18717 \\
0.19633 \\
0.21589 \\
0 \cdot 22640 \\
0.24924 \\
0.30562\end{array}$ & $\begin{array}{l}0 \cdot 388296 \\
0 \cdot 38830 \\
0 \cdot 38503 \\
0 \cdot 37975 \\
0 \cdot 37759 \\
0 \cdot 37633 \\
0 \cdot 37337 \\
0 \cdot 37164 \\
0 \cdot 36751 \\
0 \cdot 35523\end{array}$ & $\begin{array}{l}\infty \\
0 \cdot 77438 \\
0 \cdot 19664 \\
0 \cdot 16377 \\
0 \cdot 15623 \\
0 \cdot 15252 \\
0 \cdot 14518 \\
0 \cdot 14153 \\
0 \cdot 13421 \\
0 \cdot 11902\end{array}$ & $\begin{array}{l}0 \cdot 16194 \\
0 \cdot 1619 \\
0 \cdot 1495 \\
0 \cdot 1016 \\
0 \cdot 7995(-1) \\
0 \\
0 \\
0 \cdot 2403 \\
0.4310 \\
0.5504\end{array}$ & $\begin{array}{l}0.76852 \\
0.7685 \\
0 \cdot 7610 \\
0 \cdot 7639 \\
0 \cdot 7634 \\
0 \cdot 7078 / 0.8606 \\
0 \cdot 6398 / 0.1061(+1) \\
0 \cdot 1153(+1) \\
0 \cdot 1016(+1) \\
0.7343\end{array}$ \\
\hline $0 \cdot 34$ & $\begin{array}{l}0 \\
0 \cdot 18987 \\
0 \cdot 20391 \\
0 \cdot 21124 \\
0 \cdot 22265 \\
0 \cdot 22658 \\
0 \cdot 23463\end{array}$ & $\begin{array}{l}0.394380 \\
0 \cdot 38583 \\
0 \cdot 38423 \\
0 \cdot 38334 \\
0 \cdot 38187 \\
0 \cdot 38134 \\
0 \cdot 38022\end{array}$ & $\begin{array}{l}\infty \\
0 \cdot 15893 \\
0 \cdot 15338 \\
0 \cdot 15065 \\
0 \cdot 14658 \\
0 \cdot 14524 \\
0 \cdot 14256\end{array}$ & $\begin{array}{l}0 \cdot 19186 \\
0 \cdot 1304 \\
0 \cdot 8383(-1) \\
0 \\
0 \\
0 \cdot 1254 \\
0 \cdot 2827\end{array}$ & $\begin{array}{l}0.76160 \\
0.7724 \\
0.7898 \\
0.7724 / 0.8392 \\
0.6925 / 0.1040(+1) \\
0.1156(+1) \\
0.1112(+1)\end{array}$ \\
\hline 0.35 & $\begin{array}{l}0 \\
0 \cdot 78541(-4) \\
0 \cdot 76127(-1) \\
0 \cdot 20899 \\
0 \cdot 23583 \\
0 \cdot 24533 \\
0 \cdot 25515 \\
0 \cdot 27583\end{array}$ & $\begin{array}{l}0.400170 \\
0.40017 \\
0.39951 \\
0.20899 \\
0.38972 \\
0 \cdot 38868 \\
0.38756 \\
0 \cdot 38505\end{array}$ & $\begin{array}{l}\infty \\
0 \cdot 78227 \\
0 \cdot 23509 \\
0 \cdot 39237 \\
0 \cdot 14601 \\
0 \cdot 14298 \\
0 \cdot 13998 \\
0 \cdot 13407\end{array}$ & $\begin{array}{l}0.22077 \\
0.2208 \\
0.2195 \\
0.1911 \\
0.1738 \\
0.1708(-1) \\
0.4067 \\
0.5091\end{array}$ & $\begin{array}{l}0.75373 \\
0.7537 \\
0.7522 \\
0.7859 \\
0.1088(+1) \\
0.1174(+1) \\
0.1008(+1) \\
0.9151\end{array}$ \\
\hline 0.4 & $\begin{array}{l}0 \\
0.78319(-2) \\
0.68688(-1) \\
0.17769 \\
0.28875 \\
0.30799 \\
0.29328\end{array}$ & $\begin{array}{l}0.425067 \\
0.42508 \\
0 \cdot 42557 \\
0 \cdot 42792 \\
0 \cdot 43949 \\
0 \cdot 45558 \\
0 \cdot 47296\end{array}$ & $\begin{array}{l}\infty \\
0 \cdot 43669 \\
0.26392 \\
0 \cdot 18863 \\
0 \cdot 15123 \\
0 \cdot 14654 \\
0 \cdot 14969\end{array}$ & $\begin{array}{l}0.34986 \\
0.3499 \\
0.3542 \\
0.3913 \\
0.5747 \\
0.7112 \\
0.3436\end{array}$ & $\begin{array}{l}0.70317 \\
0.7032 \\
0.7039 \\
0.7074 \\
0.6924 \\
0.6083 \\
0.5366\end{array}$ \\
\hline 0.5 & $\begin{array}{l}0 \\
0 \cdot 77869(-2) \\
0.65486(-1) \\
0 \cdot 15776 \\
0.24031 \\
0 \cdot 26263 \\
0.26361 \\
0.26285\end{array}$ & $\begin{array}{l}0.458576 \\
0.45859 \\
0.45962 \\
0.46487 \\
0.47659 \\
0.48525 \\
0.48779 \\
0.48892\end{array}$ & $\begin{array}{l}\infty \\
0.48149 \\
0.31214 \\
0 \cdot 24282 \\
0 \cdot 21092 \\
0 \cdot 20473 \\
0 \cdot 20449 \\
0 \cdot 20467\end{array}$ & $\begin{array}{l}0.53591 \\
0.5360 \\
0.5448 \\
0.5958 \\
0.7247 \\
0.8446 \\
0.8971 \\
0.9293\end{array}$ & $\begin{array}{l}0.57415 \\
0.5742 \\
0.5734 \\
0.5623 \\
0.5115 \\
0.4515 \\
0.4221 \\
0.4003\end{array}$ \\
\hline
\end{tabular}

Note: Numbers in parentheses indicate powers of 10 that multiply the preceding mantissas. 


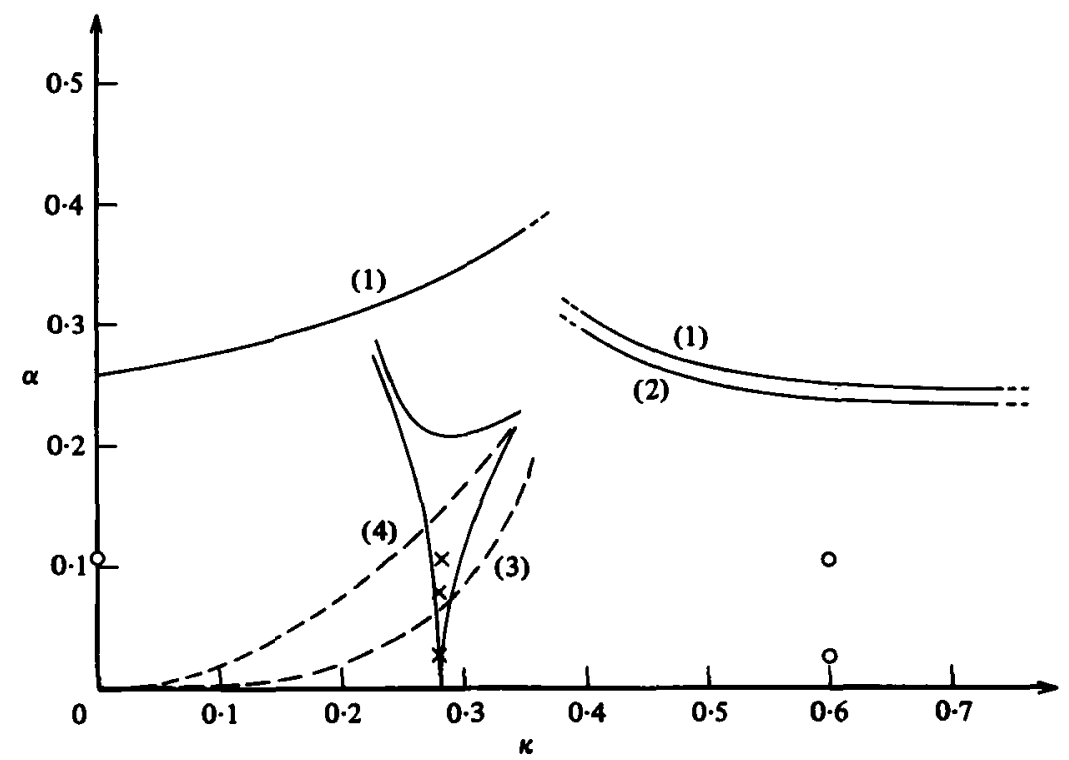

Fiaure 4. A plot of the area-spacing-ratio $(\alpha, k)$-plane. Curve 1 denotes the maximum area for a given spacing ratio. The segment corresponding to smaller $\alpha$ should be regarded as a lower bound for the maximum area because slow convergence in this region made it difficult to find the precise $\alpha$ at which vortices touched. Above curve 2, there are two solutions for a given pair $(\alpha, \kappa)$. The enclosed central region has neutral linear stability; configurations outside this region are linearly unstable. Curve 3 (see $\$ 4$ ) shows the line $E_{2}=E_{3}$, below and to the right $E_{2}>E_{3}$. Curve 4 shows the largest area for which configuration (2) exists. The symbols $\times$ mark stable and $O$ mark unstable states according to Christiansen \& Zabusky (1973).

stabilization occurs, asymptotically for small area, is approximately

$$
\left.\begin{array}{ll}
\alpha \simeq 1.31\left(\kappa_{\mathrm{c}}-\kappa\right)^{\frac{1}{t}} & \left(\kappa<\kappa_{\mathrm{c}}\right), \\
\alpha \simeq 0.78\left(\kappa-\kappa_{\mathrm{c}}\right)^{\frac{1}{2}} & \left(\kappa>\kappa_{\mathrm{c}}\right) .
\end{array}\right\}
$$

This approximate result indicates for the following reason that its exact calculation by perturbation analysis may be a laborious task. When $\alpha=0$ and $p=0.5$, the eigenvalues are the roots of the quartic

$$
\sigma^{4}+2\left(B^{2}-A^{2}\right) \sigma^{2}+\left(B^{2}+A^{2}\right)^{2}=0 .
$$

It is expected that the coefficients of the equation for the eigenvalues are analytic functions of $\kappa$ and $\alpha$. Hence, for $\alpha \ll 1$, the perturbed eigenvalues are roots of the quartic

$$
\sigma^{4}+\left[\left(2\left(B^{2}-A^{2}\right)+\alpha^{2} f_{2}(\kappa)+\alpha^{4} f_{4}(\kappa)+\ldots\right] \sigma^{2}+\left[\left(B^{2}+A^{2}\right)^{2}+\alpha^{2} g_{2}(\kappa)+\alpha^{4} g_{4}(\kappa)+\ldots\right]=0 .\right.
$$

(Invariance to changes in the sign of the vorticity requires the coefficients to be even functions of $\alpha$.) The roots will, as functions of $\alpha$, have branch-point singularities, corresponding to a change in stability of the system, where the roots of the quartic are not distinct, i.e. when

$$
-16 A^{2} B^{2}+\left[4\left(B^{2}-A^{2}\right) f_{2}-4 g_{2}\right] \alpha^{2}+\left[4\left(B^{2}-A^{2}\right) f_{4}+f_{2}^{2}-4 g_{4}\right] \alpha^{4}+\ldots=0 .
$$




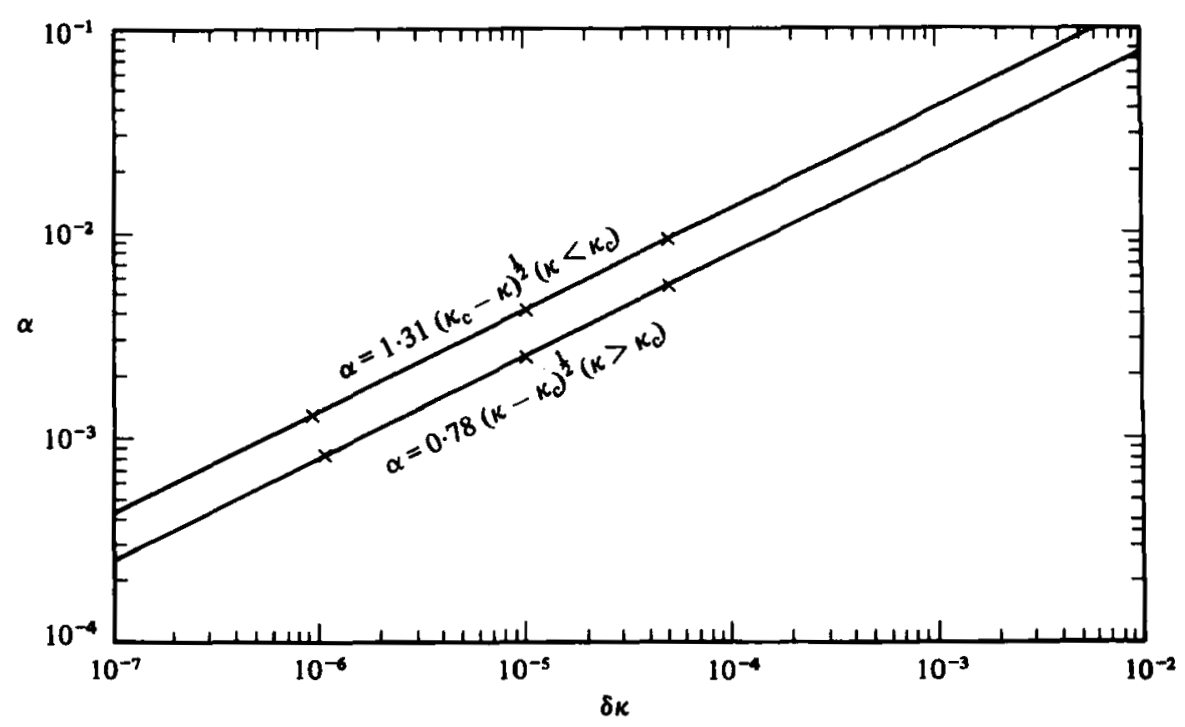

Frgure 5. A logarithmic plot of the critical area $\alpha$ at which stabilization occurs versus the distance in $\kappa$ away from $\kappa_{\mathrm{c}}=0.280549926 \ldots$.

The results shown in figure 5 indicate that, when expanded in $\kappa-\kappa_{c}$ as well as $\alpha$, this equation takes the approximate form

$$
1 \cdot 04\left(\kappa-\kappa_{\mathrm{c}}\right)^{2}-2 \cdot 32 \alpha^{2}\left(\kappa-\kappa_{\mathrm{c}}\right)+\alpha^{4}=0 .
$$

The principal point, however, is that it is necessary to go to fourth order in $\alpha$ in order to determine the behaviour of the eigenvalues for small area.

\section{The energy criterion for stability}

It was pointed out by Kelvin (1910, p. 116) (see also Arnol'd 1980, p. 335) that, for given vorticity and momentum, steady states correspond to stationary points of the kinetic energy with respect to kinematically allowable isovortical perturbations. The steady state is then stable if it is a local maximum or minimum in energy.

For this problem, assuming perturbations periodic in the streamwise direction (say with period $N l, N$ an integer), it is sufficient to apply the above criterion to one period of the flow field. Holding the vorticity constant, and assuming that vortices of opposite sense do not amalgamate, the requirement of kinematically allowable perturbations forces the total area of the vortices of each sense to remain unchanged. The condition of momentum invariance requires that the components of hydrodynamic impulse per unit length,

$$
\begin{aligned}
& I_{x}=-\frac{1}{N l} \int_{0}^{N l} \int_{-\infty}^{+\infty} \omega y d x d y=\frac{\Gamma h}{l}, \\
& I_{y}=-\frac{1}{N l} \int_{0}^{N l} \int_{-\infty}^{+\infty} \omega x d x d y=0 .
\end{aligned}
$$

stay constant. This is ensured by keeping the distance between the centroids of the two rows constant. In the following discussion it will be assumed that $N=2$. 


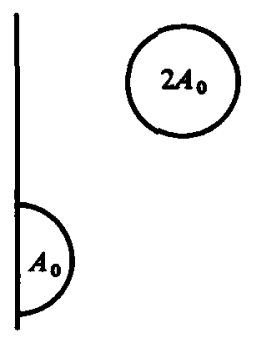

(1)

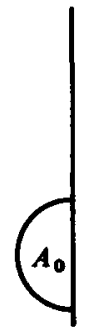

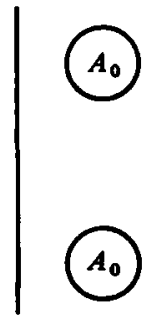

(4)

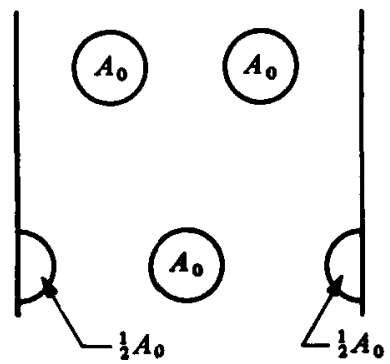

(3)
(2)
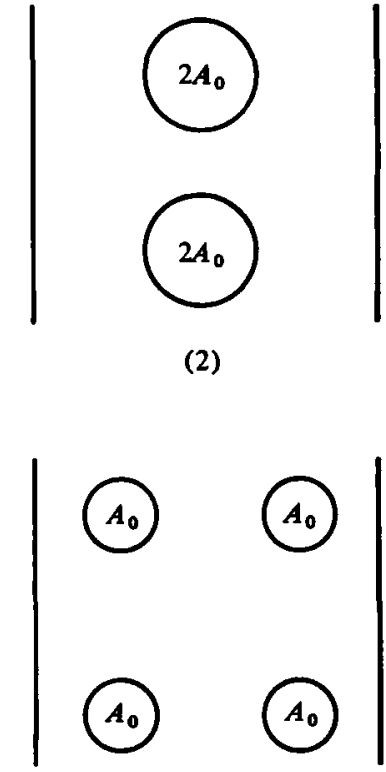

Figure 6. The four vortex configurations for the energy criterion for stability.

Now consider a system with period $2 l_{0}$ and consider the following four configurations (see figure 6):

$$
\left.\begin{array}{llll}
\text { (1) } l=2 l_{0}, & d=l_{0}, & h=h_{0}, & A=2 A_{0} ; \\
\text { (2) } l=2 l_{0}, & d=0, & h=h_{0}, & A=2 A_{0} ; \\
\text { (3) } l=l_{0}, & d=\frac{1}{2} l_{0}, & h=h_{0}, & A=A_{0} ; \\
\text { (4) } \quad l=l_{0}, & d=0, & h=h_{0}, & A=A_{0}
\end{array}\right\}
$$

These states are apparently unique for small values of $\alpha$ (below curve 2 in figure 4 for states (1) and (3)). It is clear that these four states satisfy the above conditions (4.1) and (4.2). Presumably, there are other steady states that do so, such as finer splittings and multiple-vortex layers, but the existence of such configurations is not crucial to this argument, as their energy is believed to be less than that of configuration (4) because of the general consideration that spreading out the vorticity of one sign and mixing the vorticity of opposite signs reduces the energy (Onsager 1949). We are concerned with the stability of configuration (3) to superharmonic disturbances of period $l_{0}$ in which all vortices in a row are disturbed in the same way, and to subharmonic disturbances of period $2 l_{0}$.

Calculations based on the circular-vortex approximation (Saffman \& Schatzman 1981 ) indicate that the energies of the four steady states can be ranked as follows when $\alpha=A_{0} / l_{0}^{2}$ is small:

$$
E_{1}>E_{2}>E_{3}>E_{4}
$$

Since $E$ is clearly bounded above, $E_{1}$ is an absolute maximum (since otherwise there would have to be another steady state with the same periodicity and greater energy) 
and configuration (1) is stable; it follows from the similarity of (1) and (3) that configuration (3) is stable to all superharmonic disturbances, and this will remain true for all $\alpha$ below curve 2 in figure 3 . (When there are two states, the one with less energy will be unstable.) This confirms our calculations of superharmonic instability but state (3) is not stable to subharmonic disturbances for $k$ outside the range shown in figure 4 and will therefore be in this range a minimax of energy.

Now it can be shown from calculations of the system with $d=0$ that when $\alpha$ is large it is possible for $E_{2}<E_{3}$ to occur. When this problem was first studied, it was speculated that a mechanism for the stabilization of configuration (3) against subharmonic infinitesimal and finite-amplitude disturbances is that the drop in $E_{2}$ below $E_{3}$ for sufficiently large area results in a change in the topology of the energy surface in the infinite-dimensional configuration space (for this configuration) so that it becomes a local maximum in energy. Non-dimensionalization leads to $T=l^{-1} \Gamma^{2} \hat{T}(\alpha, \kappa, \mu)$ for the energy per unit length of a given steady state (assuming it exists). The condition that $E_{2}=E_{3}$ then leads to:

$$
2 \widehat{T}\left(\frac{1}{2} \alpha, \frac{1}{2} \kappa, 0\right)=\widehat{T}\left(\alpha, \kappa, \frac{1}{2}\right),
$$

where $\alpha$ and $\kappa$ are the parameters associated with configuration (3). The result of applying this criterion is shown by curve 3 of figure 4 . It was observed that for the $\mu=0$ cases (configurations (2) and (4)), solutions exist only for vortices up to a limiting area. As the vortex area approaches this limit adjacent vortices in opposite rows approach each other. It is believed that this behaviour is qualitatively similar to that observed for a pair of counter-rotating vortices by Pierrehumbert (1980). For small $\kappa$, the limit occurs at small area, and, since interactions between neighbouring pairs are then small, the counter-rotating vortex pair should indeed be a good approximation. Curve 4 of figure 4 represents this approximation. Some calculations for the exact problem were attempted and are in reasonable agreement with curve 4 (the approximate calculated limiting areas were always less than curve 4 and within about $25 \%$ ). However, accurate calculation of the limiting area is prohibitively expensive, and was not undertaken. According to this argument, the region of stability lies between curves 3 and 4 in figure 4 and is moreover a region of stability to finite-amplitude disturbances that are not too large. The results of the linear stability analysis shows that this argument is fallacious. Incidentally, there is no evidence to suggest that the symmetrical configuration $(d=0)$ can be stabilized by finite size, but Taneda (1965) reported that oscillation of the body produced streets of symmetrical vortices. We can offer at present no explanation of this phenomenon.

\section{Conclusion}

We have generalized von Kármán's analysis of the linear stability of the point-vortex street to vortices of finite size and demonstrated that finite size can stabilize the array. The boundary of the linear-stability region for subharmonic disturbances of period twice the separation is shown in figure 4. The open questions deal with nonlinear stability and with stability to more general disturbances. At present, there seems to be no way to study the former question other than by direct numerical calculation of an initial-value problem. This was carried out to some approximation by Christiansen \& Zabusky (1973), as commented earlier, and their results indicate that linear stability 
implies nonlinear stability or, at least, very slow growth. The possibility of unstable disturbances of more general character than those considered here could also be investigated in this way.

For point vortices, the equations of motion amount to a Hamiltonian system of a finite number of degrees of freedom (depending on the assumed periodicity) and the theory of nearly integrable systems (e.g. see Chirikov 1979) suggests that this system is subject to slow instability ('Arnol'd diffusion'). However, it may be that the instability is so slow that streets of physical interest are in a practical sense stable. This question has not been resolved. Intuitively, one can perhaps expect this behaviour to persist to the finite-area case, but it would be worth while to investigate this matter further.

The importance of studying the stability to three-dimensional disturbances goes without saying, but this is a difficult problem at present.

This work was supported by the Department of Energy (Office of Basic Energy Sciences). We acknowledge with gratitude the granting of time by Control Data Corporation on the CYBER 203 computer at the C.D.C. Service Center, Arden Hills, Minnesota, where much of the more difficult computing was carried out.

\section{REFERENCES}

ARrol'D, V. I. 1980 Mathematical Methods of Classical Mechanics, pp. 332-337. Springer.

Chrrikov, B. V. 1979 A universal instability of many-dimensional oscillator systems. Phys. Rep. $52,263-379$.

Christiansen, J. P. \& ZabugkY, N. J. 1973 Instability, coalescence and fission of finite-area vortex structures. $J$. Fluid Mech. 61, 219-243.

Dомм, U. 1956 Utber die Wirbelstraßen von geringster Instabilität. Z. angew. Math. Mech. 30, 367-371.

voN KARMán, T. 1912 Utber den Mechanismus des Widerstands, den ein bewegter Körper in einer Flüsgigkeit erfährt. Gölt. Nachr. Math. Phys. Kl. 00, 547-556.

Kruvin, Lord 1910 Mathematical and Physical Papers, vol. IV. Cambridge University Press.

KochrN, N. J. 1939 On the instability of von Kármán's vortex street. C. R. Acad. Sci. U.R.S.S. 24, 19-23.

Lamb, H. 1932 Hydrodynamics, 6th edn. Cambridge University Press.

LIN, C. C. 1943 On the motion of vortices in two dimensions. University of Toronto Studies, Appl. Math. Series, vol. 5.

ONSAGer, L. 1949 Statistical hydrodynamics. Suppl. Nuovo Cim. 6, 279-287.

Nekroroshev, N. N. 1971 The behaviour of Hamiltonian systems that are close to integrable. Func. Anal. Appl. 5, 338-339.

Pierregumbert, R. T. 1980 A family of steady, translating vortex pairs with distributed vorticity. J. Fluid Mech. 99, 129-144.

Rosenhead, L. 1929 Double row of vortices with arbitrary stagger. Proc. Camb. Phil. Soc. 25, 132-138.

Safmman, P. G. \& Schatzman, J. C. 1981 Properties of a vortex street of finite vortices. SIAM J.Sci. Stat. Comp. (to be published).

TANEDA, S. 1965 Experimental investigation of vortex streets. J. Phys. Soc. Japan 20, 17141721. 